\title{
Numerical solutions of the full set of the time-dependent Nernst-Planck and Poisson equations modeling electrodiffusion in a simple ion channel
}

\author{
Ivan Valent ${ }^{1}$, Pavel Neogrády ${ }^{1}$, Igor Schreiber ${ }^{2}$ and Miloš Marek²
}

Manuscript received on July 7, 2012 / accepted on October 29, 2012

\begin{abstract}
The concept of electrodiffusion based on the Nernst-Planck equations for ionic fluxes coupled with the Poisson equation expressing relation between gradient of the electric field and the charge density is widely used in many areas of natural sciences and engineering. In contrast to the steady-state solutions of the Nernst-Planck-Poisson (abbreviated as NPP or PNP) equations, little is known about the time-dependent behavior of electrodiffusion systems. We present numerical solutions of an NPP system modeling dynamics in the interior of a membrane channel containing an electrolyte after a potential jump at one side of the membrane (voltage-clamp). The NPP equations were solved using the VLUGR2 solver based on an adaptive-grid finite-difference method with an implicit timestepping. The used approach allows for solving the full set of the NPP equations without approximations such as the electroneutrality or constant-field assumptions. Calculations reveal interesting nonlinear time evolution of the ionic concentrations and potential.
\end{abstract}

Keywords: time-dependent Nernst-Planck and Poisson equations, electrodiffusion, PNP theory, nonlinear dynamics, membrane, ion channel, voltage-clamp.

\section{INTRODUCTION}

Movement of charged particles driven by gradients of both chemical and electrical (i.e. electrochemical) potentials is termed an electrodiffusion. The corresponding flux defined as the number (in moles) of the particles transported per unit time through unit area is given by the Nernst-Planck equation:

$$
J_{i}=-D_{i}\left(\nabla c_{i}+\frac{z_{i} F}{R T} c_{i} \nabla \varphi\right)
$$

where $J_{i}$ denotes the flux of $i$-th particle, $c_{i}$ and $z_{i}$ are the molar concentration and the charge number, respectively, and $\varphi$ is the electric potential. The symbols $F$ and $R$ stand for the Fara- day and universal gas constants, respectively, and $T$ represent the absolute temperature.

In general, movement of charge is accompanied by a change of the electric field due to a change in the charge density. The charge density $\rho$ (in $\mathrm{C} \mathrm{m}^{-3}$ ) is related to the electric field intensity $E$ by the Poisson equation:

$$
\nabla E=\frac{\rho}{\varepsilon}
$$

where $\varepsilon$ is the permittivity of the medium.

The Nernst-Planck equations describing the fluxes of individual particles (e.g. ions) coupled to the field Poisson equation represent a mathematical framework for description of the elec-

Correspondence to: Ivan Valent - E-mail: valent@fns.uniba.sk

1 Department of Physical and Theoretical Chemistry, Faculty of Natural Sciences, Comenius University, Mlynská dolina CH1-327, 84215 Bratislava, Slovak Republic

${ }^{2}$ Department of Chemical Engineering, Institute of Chemical Technology, Prague, Technická 5, 16628 Prague 6, Czech Republic 
trodiffusion under the assumption of continuity usually referred to as the Nernst-Planck-Poisson (NPP) theory. The NPP theory (in the biophysics field also referred to as PNP theory) provides a valuable tool for investigation, analysis, modeling, and general theoretical description aimed at elucidation and interpretation of electrodiffusion phenomena that may be encountered in many areas of both living and non-living matter. The concept of electrodiffusion based on the NPP theory extends to a variety of fields such as membrane biophysics, enzyme kinetics, colloid science, electrochemistry and electroanalytical chemistry, microfluidics, solid state physics, and materials science. Although the mathematical formulation of the NPP theory is expressed in an elegant compact form of a set of partial differential equations, to solve these nonlinear equations is a particularly difficult task. Since the classical papers by Planck [1] and Goldman [2], much work has been done on finding steady-state solutions of the NPP equations, e.g. modeling of ion transport through liquid junctions [3], protein channels $[4,5]$, and synthetic nanopores $[6,7]$. However, very little is known about the time-dependent behavior of electrodiffusion systems.

An early attempt to solve nonlinear time-dependent NPP equations was presented in 1958 by Helfferich \& Plesset [8], who solved the electrodiffusion equations applied to ion exchange kinetics assuming the electroneutrality condition introduced by Planck. Using the finite differencing they obtained numerical results for counter ions of equal valence and different mobilities.

In 1965 in their renowned work, Cohen \& Cooley [9] showed for the first time numerical solutions of the time-dependent NPP equations for a thin permeable membrane between electrolytic solutions $\left(\mathrm{Na}^{+} / \mathrm{H}^{+} / \mathrm{Cl}^{-}\right)$. The time and space changes in concentration, electrical potential and effective conductance were calculated as the response of the system to step current input. The current was defined including contribution of the displacement current. Finite-difference approximations were used for the space and time derivatives and a predictor-corrector scheme was used for time integration. Charge neutrality was assumed for the time-dependent case with additional discussion of boundary effects that occur when charge neutrality does not hold in the steady-state case.

Hafemann [10] then presented a method for computation of the liquid junction potential as a function of time and discussed applications to more complex systems, including biological membranes.

Particularly important is a contribution of MacGillivray [11] who solved analytically the time-dependent NPP system applied to a biological membrane. He introduced boundary conditions reflecting a "voltage-clamp" type of experiment, in which the system is subject to an instantaneous jump in potential difference across the membrane. The solutions were given in the form of asymptotic expansions, valid for small and large times and small voltage-clamp values. These results showed that the early time perturbation concentrations do not satisfy the electroneutrality assumption.

Another work analyzing time-dependent behavior of a membrane under the voltage clamp conditions is due to Offner [12]. He developed a numerical technique employing Crank-Nicolson method to calculate ionic current flows following a depolarizing step. Alternatively to the Nernst-Planck formulation of the problem, the activation energy formulation of diffusion was applied to the diffusion process treated as a series of discrete jumps across potential barriers.

Brumleve \& Buck [13] presented an efficient simulation procedure for the steady state and transient solution of the NPP equations. The fully implicit algorithm involving Newton-Raphson technique allowed treatment of multi-ion systems and included refinements in time and distance scaling ensuring computational efficiency. The effectiveness of their method was demonstrated by using examples from the fields of membrane electrochemistry and solid state physics. The procedure allows for simulation of a transient response of the system to current or charge steps or ramps. However, potential is a dependent variable and cannot be controlled.

The work of Brumleve \& Buck was followed by others, for example, the approach of numerically solving the NPP equations was used to study equilibrium and nonequilibrium diffuse electrical double layer at the metal electrode/electrolyte solution interface [14] and in ion-exchange membranes [15]. Rudolph [16] simulated electrical migration and diffuse double-layer effects using a fast implicit finite difference algorithm, which was developed as a part of a general tool for modeling electrochemical processes related to electroanalytical chemistry. Liquid junction and ion selective membrane potentials were studied using a numerical model based on the time-dependent NPP equations by Sokalski et al. [17]. Bieniasz [18] extended his numerical technique based on the finite-difference patch-adaptive strategy to time-dependent models involving electrodiffusion transport described by NPP equation systems in one-dimensional space geometry. Lim et al. [19] presented a finite element implementation of the NPP and modified NPP models for a transient analysis of electric double layer. Examples of recent applications of the 
NPP theory to time-dependent problems concern translocation of DNA through nanopores and nanochannels [20], or electrochemical methods like cyclic voltammetry [21] and electrochemical impedance spectroscopy [22].

Numerical methods used for solving an initial boundary value problem of the NPP equation system can be divided into three groups. Most frequently used is the finite-difference approach as documented by majority of the references cited above. The second group is represented by techniques based on the finite element method. Although implementations of this method for time-dependent electrodiffusion problems were rare a decade ago [23], recent work [19, 24, 25, 21] indicates advancements. A special class among the numerical methods used to solve the NPP equations in space and time represents the "network thermodynamic method" introduced by Horno et al. [26]. The key strategy of this approach is to transform the governing partial differential equations into an equivalent electric circuit and to solve numerically the resulting set of ordinary differential equations in time. Most of the works mentioned above used $1 \mathrm{D}$ geometry. Implementations of models in higher spatial dimensions for the time-dependent NPP problems are scarce. Samson et al. [27] published an interesting work on modeling electrodiffusion mechanisms in porous media in $1 \mathrm{D}$ and $2 \mathrm{D}$ geometries. Finite-difference methods used for initial value problems involving the NPP equations cover a wide spectrum of algorithms between entirely explicit and entirely implicit techniques. Although explicit algorithms are more convenient for implementation, they were criticized for numerical instability and computational ineffectiveness. Time integration schemes used by implicit methods usually include the implicit Euler scheme and backward differentiation formulae (BDF). Most of the finite-difference implementations that can be found in the literature concerning the NPP systems used fixed spatial grids and time steps in contrast to rare application of more efficient adaptive methods. A detailed review of the finite-difference approaches to solving the time-dependent NPP equations can be found in [18].

Apart from numerical methods, significant results have been obtained by analytical approach to finding solutions of the timedependent NPP equations. From many contributions we mention here works of Bazant et al. [28, 29], who analyzed the diffusecharge dynamics in electrochemical systems using matched asymptotic expansions. Of interest is also an investigation of charge transport in a planar device on application of a voltage step by Beunis et al. [30], and an analysis by Golovnev \& Trimper [31] giving an exact solution to the time-dependent NPP equa- tions in the linear regime for a polyelectrolyte system subjected to a dc applied voltage.

Our motivation is a perspective of modeling electrodiffusion dynamics in biological processes. This aim requires implementation of numerical techniques of solving the NPP equations for complex models with spatially $2 \mathrm{D}$ and $3 \mathrm{D}$ geometry. We started with a simple two-dimensional model of a membrane channel and simulated concentration of ions and electric potential in space and time after a voltage or current step using the VLUGR2 solver for time-dependent PDEs in 2D [32]. The problem was formulated to be independent on $y$-coordinate, so the obtained numerical solutions are comparable to the analytical limits for 1D steady-state solutions and to the $1 \mathrm{D}$ asymptotic solutions for early times. In addition to testing the 2D solver for applicability to solving the time-dependent NPP equations, the results reported here bring further insight into physical processes of charge separation in a solution of strong electrolyte in a membrane under the voltageclamp conditions. Although the one-dimensional problem under study is classical, to our knowledge, spatiotemporal profiles of ionic concentrations, fluxes, electric potential, and both the faradaic and the displacement currents have not been presented in the literature for the given formulation without approximations such as those arising from the electroneutrality or the constant field assumptions.

\section{THEORY}

The used biophysical model is represented by a rectangular domain of $10 \mathrm{~nm}$ length and $3 \mathrm{~nm}$ height describing a simple membrane channel. The interior of the domain was filled by a water solution of a uni-univalent strong electrolyte without any internal or boundary fixed charges. The lower and upper boundary of the domain carried the Neumann boundary condition for the ionic concentrations and the potential: $\partial c / \partial y=0, \partial \varphi / \partial y=0$. Also, the initial and side boundary conditions were formulated without any gradients in $y$-direction, so the analyzed problem may be treated as one-dimensional. This simplification makes it possible to compare solutions obtained by a 2D solver of PDEs with the existing solutions and to test reliability and applicability of the chosen solver to deal with the electrodiffusion problems defined in two and potentially three dimensions. The left $(x=0)$ and right $(x=10 \mathrm{~nm})$ boundaries were kept at constant concentrations of the electrolyte of 50 and $500 \mathrm{mmol} \mathrm{dm}^{-3}$, respectively. The left boundary was all the time grounded at zero potential, while the right one was subject to a sudden (at $t=0$ ) rise of the potential from zero to a value of $+100 \mathrm{mV}$. 
Initial concentrations of cation and anion were equal to each other with linear distribution along the $x$-coordinate, conforming to the constant gradient across the membrane given by the boundary conditions. Time evolutions of both ion concentrations and of electric potential after the potential change were calculated by numerical solving the governing PDEs. The conditions specified above are referred to as standard and were used in most of calculations. Alternatively to the potential step (voltage clamp), the current step condition were also applied. The used biophysical model was inspired by a model of the gramicidine A channel [5] with some modification and simplification. The channel length was extended to $10 \mathrm{~nm}$ which is a usual value representing the membrane thickness.

The governing equations were those arising from the NPP theory. For the non-stationary problems the fluxes and concentrations obey the continuity equation:

$$
\frac{\partial c_{i}}{\partial t}=-\nabla J_{i}
$$

where $t$ is time. Considering that the electric field intensity is related to the electric potential

$$
E=-\nabla \varphi
$$

and applying the continuity equation on the Nernst-Planck equation (1) for both ion fluxes, the following expressions are obtained for time derivative of ionic concentrations:

$$
\begin{aligned}
& \frac{\partial c_{1}}{\partial t}=D_{1}\left[\nabla^{2} c_{1}+\frac{F}{R T}\left(\nabla c_{1} \nabla \varphi+c_{1} \nabla^{2} \varphi\right)\right] \\
& \frac{\partial c_{2}}{\partial t}=D_{2}\left[\nabla^{2} c_{2}-\frac{F}{R T}\left(\nabla c_{2} \nabla \varphi+c_{2} \nabla^{2} \varphi\right)\right]
\end{aligned}
$$

where the indexes 1 and 2 are related to cation and anion, respectively. Expressing the charge density in the equation (2) by means of the ion concentrations and with respect to (4), the Poisson equation takes the form:

$$
\nabla^{2} \varphi=-\frac{F}{\varepsilon_{0} \varepsilon_{r}}\left(c_{1}-c_{2}\right)
$$

where $\varepsilon_{0}$ and $\varepsilon_{r}$ are the vacuum permittivity and the relative permittivity of the medium, respectively. The initial boundary value problem described by equations (5)-(7) was solved with the initial and boundary conditions as described above, using the following standard set of the parameters (unless otherwise noted): $D_{1}=1 \times 10^{-6}, D_{2}=5 \times 10^{-7} \mathrm{~cm}^{2} \mathrm{~s}^{-1}, T=293 \mathrm{~K}$, and $\varepsilon_{r}=80$. The parameter values were taken from [5] with minor modifications. The diffusion coefficient of anion was decreased twice to demonstrate more general example of inequal ionic mobilities. For the step-current case the left boundary was also held at zero potential, whereas the right boundary condition, instead of the Dirichlet one for the voltage-clamp case, was prescribed as follows:

$$
D_{1}\left(\frac{\partial c_{1}}{\partial x}+\frac{F}{R T} c_{1} \frac{\partial \varphi}{\partial x}\right)-D_{2}\left(\frac{\partial c_{2}}{\partial x}-\frac{F}{R T} c_{2} \frac{\partial \varphi}{\partial x}\right)=-\frac{i_{F}}{F}
$$

where $i_{F}$ denotes the imposed faradaic current density.

Calculated spatiotemporal profiles of the ion concentrations and the potential may be used to evaluate other quantities of interest by an a posteriori numerical derivation as described in the next section. The ion fluxes and their decomposition to diffusion and migration terms were determined according to equation (1). The ionic fluxes may be subsequently used to figure out the faradaic current caused by the net charge flow through the channel. The electric field $E$ was evaluated using its relation to the electric potential (Eq. 4), ignoring electrodynamical contribution from the change of magnetic field in time. However, time change of the electric field induces the displacement current, whose contribution to the total current may be significant in electrolyte solutions $[9,14,33]$. So the total current density was calculated as the sum of the faradaic (conducting) current and the displacement current in accordance with the Maxwell's definition of "the true electric current" [34]:

$$
i_{T}=i_{F}+i_{D}=i_{F}+\varepsilon \frac{\partial E}{\partial t}=i_{F}-\varepsilon_{0} \varepsilon_{r} \frac{\partial^{2} \varphi}{\partial x \partial t}
$$

where $i_{T}$ and $i_{D}$ stand for the total and displacement current densities, respectively. The second mixed spatio-temporal derivative of the potential was used for the displacement current evaluation.

\section{COMPUTATIONAL METHODS}

The system of the time-dependent NPP equations (5)-(7) was solved with the use of an adaptive grid solver for PDEs in 2D called by the autors VLUGR2 [32]. This Fortran 77 code is based on an adaptive-grid finite-difference method with an implicit timestepping. In the following, we present a brief outline of the numerical approaches utilized by the solver. More detailed information about algorithmic aspects and the code structure of the VLUGR2 can be found in [32].

The concept of a Local Uniform Grid Refinement method lies on adaptive grid refinement in regions with high spatial activity. 
Starting from a uniform base grid covering the whole domain, a nested finer subgrid is recursively created where required by division of a coarser grid cell in 4 equal parts. The location and size of the subgrids are readjusted at each time step. For the space discretization, standard second-order finite differences are used, central on the internal domain and one-sided at the boundaries. The refinement is governed by a curvature monitor SPCMON:

$$
\begin{gathered}
\operatorname{SPCMON}(i, j):=\max _{i c=1, \mathrm{NPDE}} \operatorname{SPCTOL}(i c) \\
\times\left(\left|\Delta x^{2} u_{x x}^{i c}(i, j)\right|+\left|\Delta y^{2} u_{y y}^{i c}(i, j)\right|\right)
\end{gathered}
$$

where $(i, j)$ is a specific grid point and $i c$ denotes a component of the solution $u$ of the number NPDE of PDEs. The subscripts $x x$ and $y y$ mean second partial derivatives with respect to $x$ - and $y$-coordinate, respectively. $\Delta x$ and $\Delta y$ are the grid width in the $x$ - and $y$-direction, respectively, and

$$
\operatorname{SPCTOL}(i c):=\frac{\operatorname{SPCWGT}(i c)}{\operatorname{UMAX}(i c) \mathrm{TOLS}}
$$

The variables SPCWGT, UMAX, and TOLS are user specified quantities - a weighting factor for the relative importance of a PDE component on the space monitor, the approximate maximum absolute value of a component, and the space tolerance, respectively.

The system of PDEs is solved by the VLUGR2 using the Method of Lines (MoL) approach. After the space discretization of the PDEs a system of ordinary differential equations (ODEs) or differential algebraic equations (DAEs) is obtained. The resulting system of ODEs/DAEs is integrated in time using the secondorder two-step implicit Backward Differentiation Formula (BDF) with variable step size. In the first step the Backward Euler method is used. The time integration is controlled by means of both absolute and relative tolerances which depend on the user specified parameters UMAX and TOLT. A relative weighting factor TIMWGT may be also specified. Although the size of each integration time step is automatically adapted by the solver, the user may control its lower and upper bound by DTMIN, DTMAX parameters, respectively. The initial time step size DT needs to be specified directly by the user.

We used a double precision version of the VLUGR2 code. For a standard calculation the domain was covered by a basic grid of $10 \times 3$ cells in $x$ and $y$ direction, respectively, with grid refinement allowed up to level 4 . The initial time step size DT = $10^{-11} s$ was used and space and time tolerances were specified as TOLS $=0.005$ and TOLT $=0.003$. The default option BiCGStab with ILU preconditioning was used as linear solver. Jacobian matrix was computed by numerical differencing. Convergence was tested by comparing the results obtained with various grid densities and time step sizes. Computations were carried out on a PC equipped by $2.13 \mathrm{GHz}$ Intel Xeon 3050 processor. Duration of a job did not exceed several seconds.

The ion fluxes, currents and electric field were calculated by an a posteriori differentiation of the obtained solution profiles using the expressions described in the previous section. Numerical evaluations of the first and diagonal second derivatives $\left(\partial^{2} / \partial x^{2}\right)$ were obtained using seven (generally nonequidistant) point differential quadrature-like scheme which supress the contaminantion from higher (up to 6th) order derivatives. We examined several other computational setups (from the common 3point formulae up to 11-point scheme), but the 7-point approach with properly adjusted distances of the independent variable gave most stable and reliable results. Accuracy of the calculated mixed spatio-temporal second derivatives $\left(\partial^{2} / \partial x \partial t\right)$ was verified by comparison of the $\partial(\partial / \partial t) / \partial x$ and $\partial(\partial / \partial x) / \partial t$ values. The numerical partial derivatives also allow for testing the obtained solutions. For the purposes of the a posteriori data processing the solution profiles were calculated on a fixed uniform grid of $160 \times 3$ cells giving resolution $\Delta x=0.0625 \mathrm{~nm}$ without further refinement.

\section{RESULTS AND DISCUSSION}

\subsection{Tests of accuracy of the numerical steady-state solutions}

A basic test of reliability of the adopted numerical technique for solving the time-dependent NPP equations provides the possibility of comparing the calculated steady-state profiles with the limit solutions available in the form of analytical expressions. As mentioned above, the electroneutrality assumption, first used by Planck [1], is applicable to long channels in contrast to the constant-field approximation, used by Goldman [2], which is valid for short channels. Analytical expressions for the ion concentration and the electric potential profiles for both cases referred to as the "long-" or the "short-channel limit" can be found in the basic literature [35].

We compared steady-state profiles calculated under various conditions with the analytical limits. The results confirmed that the numerical solution for low electrolyte concentrations approach the short-channel limit with decreasing the channel length, while at higher ionic concentrations and longer channels the electroneutrality condition becomes valid and the solutions are close to the long-channel limit. Figure 1 illustrates the testing calculations with the standard set of parameters. The calculated steady-state 
profiles of the ionic concentrations through the membrane for the full NPP system (circles) slightly differ from the straight line demonstrating a moderate deviation from electroneutrality which gradually vanishes with the increasing channel length. On the other hand, shortening the channel induces a more significant separation of the cationic and anionic concentration profiles towards the nonlinear limits given by the constant-field assumption. The constant-field condition may be imposed for an arbitrary length of the channel by setting the right-hand side of the Poisson equation (7) to zero. The numerical stationary solution of the NPP system with the Poisson equation fixed that way (boxes) fully reproduces the short-channel limit represented by the well-known Goldman-Hodgkin-Katz equations.

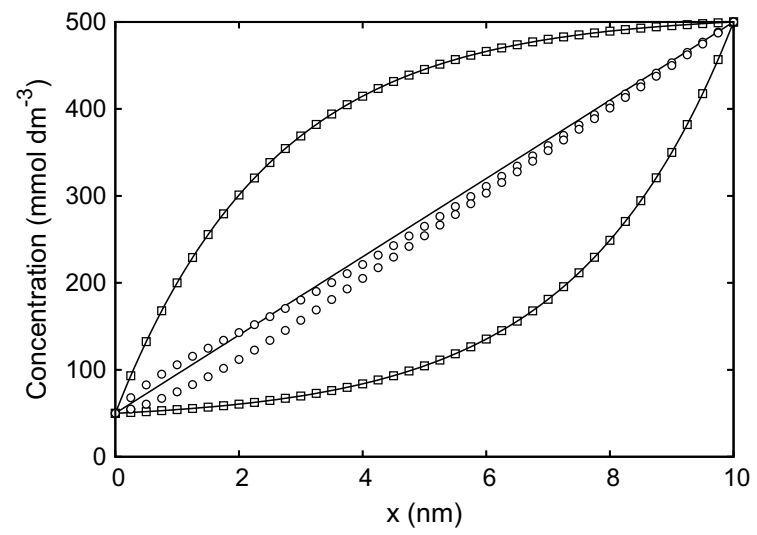

Figure 1 - Comparison of the calculated steady-state concentration profiles with the analytical limit solutions. The solid lines represent the theoretical profiles corresponding to the electroneutrality (the straight line - long-channel limit) and the constant-field (the curved lines - short-channel limit) assumptions. The circles and boxes indicate the calculated ionic concentrations with the full set of the NPP equations and with the imposed constant field (see text), respectively. The upper and lower lines or points correspond to the cationic and anion concentrations, respectively. The standard conditions (see the section Theory) were used in the calculations.

Numerical steady-state solutions of the NPP equations for the conditions corresponding to intermediate regions out of the applicability of both the analytical limits are available in the literature. We compared our solution with that presented by Corry and coworkers [36] for a $2.5 \mathrm{~nm}$ long channel with the concentrations of the electrolyte maintained at 500 and $100 \mathrm{mmol} \mathrm{dm}^{-3}$ at the left and right end, respectively, under the potential difference of $100 \mathrm{mV}$ through the channel. The solutions matched very well, although small differences in the ionic concentrations appeared, particularly in the central region of the channel. This effect may be attributed to the different definition of the channel boundary for the systems under comparison. Corry et al. used a cylindrical 3D model of the ion channel with the solid wall and they obtained the $1 \mathrm{D}$ solution as a convergence limit for the increasing diameter of the channel. We used a 2D model as previously described giving a solution which was proved to be independent on the $y$ coordinate in accordance with the problem definition.

\subsection{Comparison of the time-dependent numerical and asymptotic analytical solutions}

Existence of an asymptotic analytical solution of the time-dependent NPP equations found by MacGillivray [11] for early times and small voltage-clamp values makes it possible to test the used numerical approach with respect to temporal dynamics and discuss time evolution of both the solutions for larger times. To this end, we performed calculations under the conditions that allow direct comparison of the numerical and analytical solutions. The diffusion coefficients of cation and anion were increased from their standard values to $D_{1}=2 \times 10^{-5}$ and $D_{2}=1 \times 10^{-5} \mathrm{~cm}^{2} \mathrm{~s}^{-1}$. The boundary electrolyte concentrations were set to 100 and $200 \mathrm{mmol} \mathrm{dm}^{-3}$ for the left and right boundary, respectively. The right boundary potential was subject to a voltage step (at the time $t=0$ ) from the initial value of $50 \mathrm{mV}$ to $52.5 \mathrm{mV}$. The initial spatial profiles of the ion concentrations and the potential were set to functions representing the steady-state solutions of the NPP equations satisfying the boundary conditions at $t=0$. Since the electroneutrality assumption is not quite fulfilled, analytical solutions could not be used as the initial profiles. The profile functions were obtained in a preliminary calculation by solving the time-dependent problem of the voltage step from zero potential to a given value $(50 \mathrm{mV})$ with linear initial distribution of ion concentrations. After a time long enough to reach the steady state, the integration was stopped and the solution was used as the initial conditions for the main calculation. Other model parameters were the same as those specified in the Theory section for the standard simulation.

The resulting solution is presented in the form of the perturbation variables which describe time evolution of the difference between the actual value of a dependent variable (ion concentration or potential) and its initial steady-state value. Figure 2 shows perturbation concentrations of ions as functions of time and position in the channel using the dimensionless variables introduced by MacGillivray [11]. It can be seen that the numerical solution for both ions initially follows the asymptotic limits. The analytical solutions for various positions coincide at early times, so only the two solid lines for each ion can be distinguished in Figure 2a. For the dimensionless time $\tilde{t}>0.01$ the numerical solutions markedly diverge from each other depending on 
the dimensionless position $\tilde{x}$ and significantly deflect from the asymptotes. This is mainly due to the fact that validity of the analytical solution is restricted for "small time" satisfying the condition $\tilde{t} \ll \alpha^{2}=\varepsilon R T /\left(F^{2} L^{2} C\right)$, where $L$ is the thickness of the membrane (the channel length), $C$ is the concentration at $x=0$, and other parameters have the same meaning as previously explained. For the conditions used in the calculation $\alpha^{2}=0.0185$, which is in agreement with the discussed observations. Already at early times the process of charge separation occurs demonstrated by a positive growth of the perturbation concentration of cation in contrast to the perturbation concentration of anion which declines to negative values. This confirms the fact pointed out by MacGillivray that the early time perturbation concentrations do not satisfy an electroneutrality assumption. At higher times the nonlinear effects become significant resulting in non-monotonous evolution of the cation perturbation concentration which passes through a maximum. The steady state is established approximately at $\tilde{t} \approx 2$.

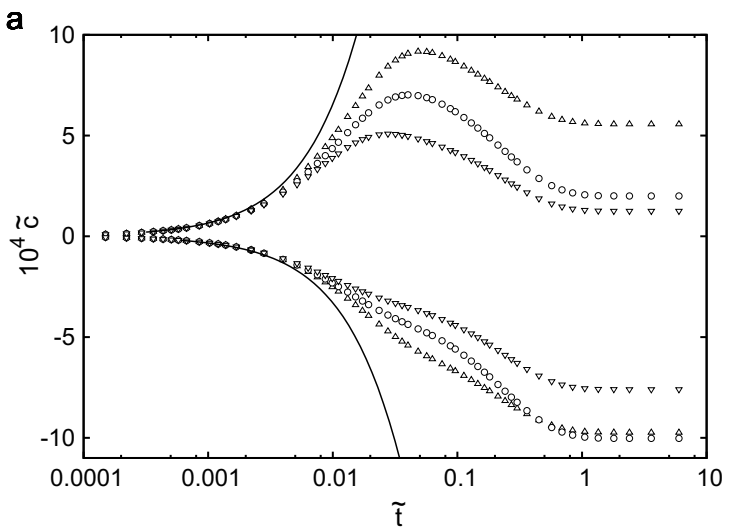

b

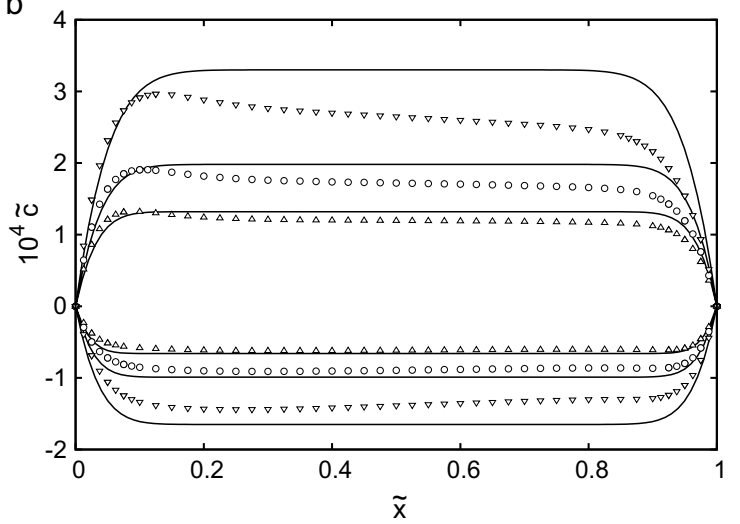

Figure 2 - The dimensionless perturbation concentration of cation (upper lines) and anion (lower lines) as functions of the dimensionless time $\tilde{t}$ (a) and the dimensionless position $\tilde{x}$ (b). The solid lines represent MacGillivray's [11] asymptotic solutions. The upper triangles, circles, and lower triangles correspond to the numerical solution: (a) at $\tilde{x}=0.25,0.5$, and 0.75 respectively; (b) at $\tilde{t}=0.002,0.003$, and 0.005 , respectively. See the text for details.
The spatial profiles shown in Figure $2 b$ also demonstrate good agreement between the numerical and analytical solutions at early times $(\tilde{t}=0.002$ and 0.003$)$, in particular for the anion perturbation concentration. With increasing time, apart from the deviation from the asymptotes, the numerical solution continuously loses its symmetry across the channel center with a more profound effect of the charge separation at the left boundary.

An additional source of discrepancy between the presented numerical solution and the analytical solution given by MacGilliray is the definition of the initial (and hence the final) steadystate condition. MacGillivray constructing his asymptotic expansions [11] used the solution corresponding to electroneutrality, noting that other approximations, such as constant field assumption, could also be used to describe the quiescent state. Once the electroneutrality assumption is applied as the initial condition, the final steady state will also satisfy electroneutrality as MacGillivray proved by analysis of the "large time" behavior [11]. Thus, the perturbation concentration of ions initially stirred by the voltage jump will return to zero values when the new steady state is achieved. In fact, for a channel of a finite length, the electroneutrality solution is not a true solution of the stationary NPP equations, rather it is a limit of the actual steady-state solution for a channel length approaching infinity. Nevertheless, the electroneutrality assumption is a good approximation applicable for channels of more than, say, $10 \mathrm{~nm}$ length assuming usual electrolyte concentrations. However, even for relatively long channels, the steady-state concentration profiles of ions will curve from linearity in a non-zero electric field. Consequently, in a real channel, the final steady state will differ from the initial one not only in the profile of the potential, but also in distribution of the ion concentrations, even for small voltage-clamp values. Accordingly, the perturbation concentrations in Figure 2a do not converge to zero, but are split into several final values depending on the sign of ion charge and the position $\tilde{x}$.

\subsection{The time-dependent behavior of the NPP system under the voltage/current-clamp}

In this section we present the numerical results of solving the time-dependent NPP system under the conditions specified above (the standard conditions) using the computational methodology based on the VLUGR2 solver for PDES as previously described. Figure 3 shows the time evolution of the ionic concentrations and the electric potential at one quarter of the channel $(x=2.5 \mathrm{~nm})$ after a potential jump from 0 to $+100 \mathrm{mV}$. The figure illustrates typical nonlinear dynamics of the process which is extremely fast 
at the beginning requiring a very small and flexible time step. After a nanosecond, the charge separation is obvious (Fig. 3a) and the ionic concentrations diverge from each other. While the concentration of cations passes through a maximum at roughly $20 \mathrm{~ns}$, the anionic concentration exhibits a sigmoid decrease. The steady state is established after approximately $1 \mu \mathrm{s}$ from the voltage switch-on and apparently does not satisfy electroneutrality. The time course of the potential (Fig. 3b) shows a sigmoid increase from the initial value of $25 \mathrm{mV}$ to a steady-state value of $47.4 \mathrm{mV}$. Although the initial conditions prescribe the zero potential as the starting value everywhere in the channel, after the first time step the potential profile is abruptly switched by the solver to a linear one, correctly satisfying the new potential boundary condition and the initial electroneutrality. Subsequently, the electric field, and the potential accordingly, is continuously changed as the ions move in the process of electrodiffusion.

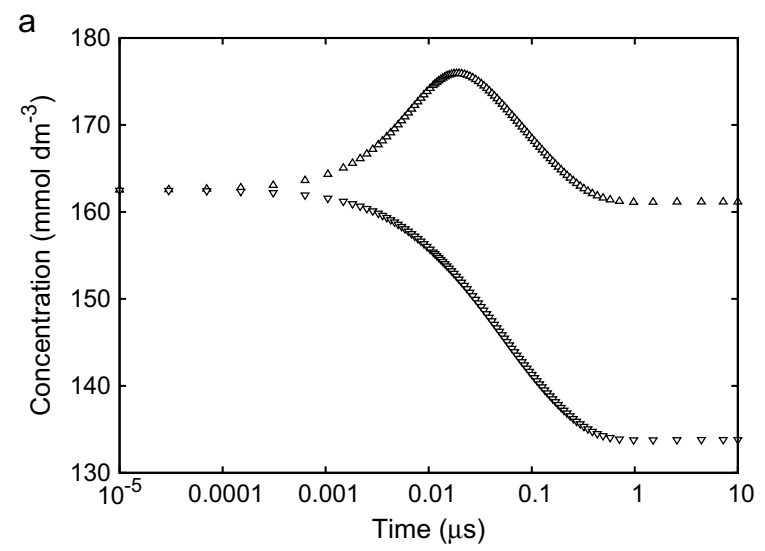

b

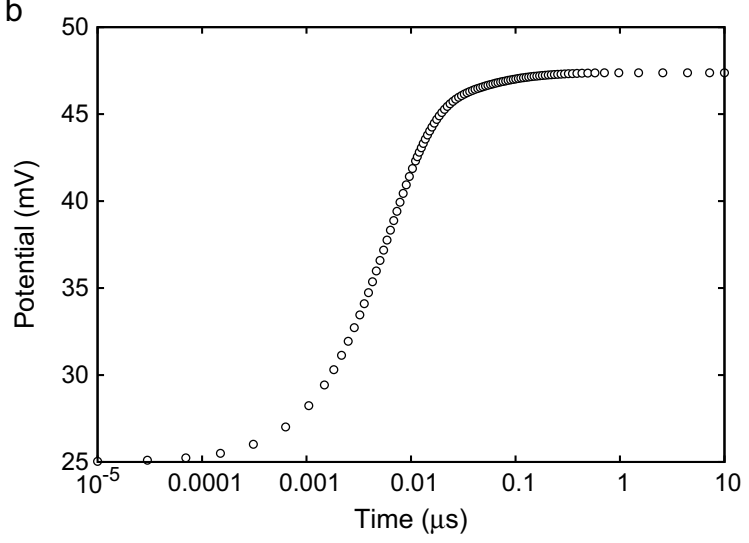

Figure 3 - Time courses (at $x=2.5 \mathrm{~nm}$ ) of the ionic concentrations (a) and the electric potential (b) for the voltage-clamp numerical simulation. The upper and lower triangles denote the concentration of cations and anions, respectively. The standard conditions (see the section Theory) were used in the calculation.

Applying voltage-step of the negative polarity leads to the same time courses of the ionic concentration, however cationic and anionic course are exchanged. By increasing the anionic diffusion coefficient twice, so that its value equals that for the cation, causes a shift of the maximum in the cationic concentration course to an earlier time associated with a decrease of the maximum value. The inflection point of the anionic concentration and the potential curves show a similar shift to shorter times. Further increase of the anionic diffusion coefficient exceeding the cationic value makes the described effect more pronounced.

The presented standard calculation with the tolerances set as TOLS $=0.005$ and TOLT $=0.003$ required 99 various time steps with the dynamically adapted space grid as documented by Figure 4. The most densely spaced grid of the fourth level allowing a resolution of $\Delta x=0.125 \mathrm{~nm}$ was generated after $10 \mathrm{~ns}$ and covered almost one half of the computational domain indicating a higher spatial activity in the left part of the channel.

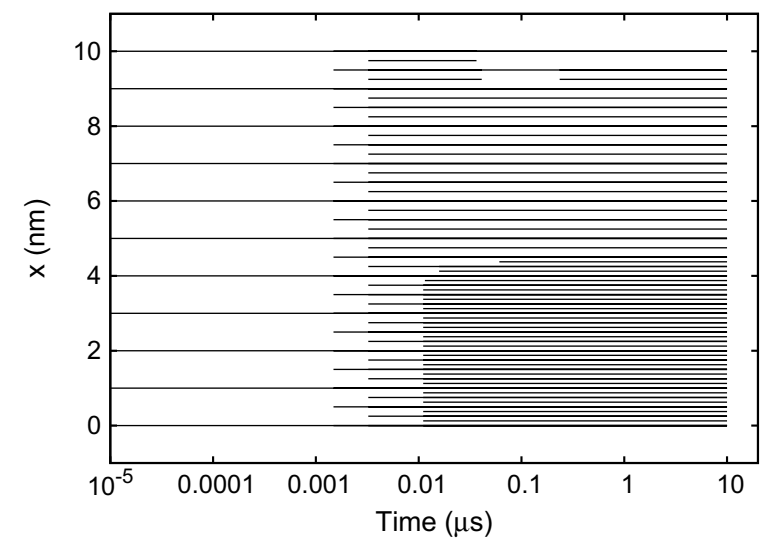

Figure 4 - Space grids generated in the course of the standard calculation (see Fig. 3). Only the $x$-nodes are shown, while the $y$-nodes were kept fixed.

The space tolerance TOLS affects the computational accuracy influencing the grid refinement by means of the curvature monitor (Eqs. 10,11), however, once the spatial resolution is set on by the grid adjustment, or the fixed grid is used, the TOLS parameter has no impact on the effective accuracy of the calculation. The time tolerance TOLT is particularly important for the problem under study and a proper specification of this parameter is crucial to obtain correct results. The effect of the TOLT parameter on the residuals - differences of the right-hand and left-hand sides of the solved equations (5)-(7) and on the calculated values of the ionic concentrations and the potential was investigated using the standard NPP model as previously specified. Table 1 lists the residuals and the values of the dependent variables for $x=7 \mathrm{~nm}$ at $t=10 \mathrm{~ns}$. The numbers of the time steps performed for the whole calculations to the end time of $10 \mu \mathrm{s}$ for the given TOLT values are also included. It can be seen that a decrease of TOLT 
Table 1 - The residuals and the calculated ionic concentrations for $x=7 \mathrm{~nm}$ at $t=10 \mathrm{~ns}$. The fixed spatial grid of $80 \times 4$ cells with TOLS $=0.005$ and the standard set of other parameters were used in the calculation. The residuals RES1, RES2, and RES3 correspond to the difference of the right and left side of eqs. 5, 6, and 7, respectively.

\begin{tabular}{|c|c|c|c|c|c|c|c|}
\hline TOLT & $\begin{array}{l}\text { Time } \\
\text { steps }\end{array}$ & $\begin{array}{c}\text { RES1 } \\
{\left[\mathrm{mM} \mathrm{ms}^{-1}\right]^{a}}\end{array}$ & $\begin{array}{c}\text { RES2 } \\
{\left[\mathrm{mM} \mathrm{ms}^{-1}\right]}\end{array}$ & $\begin{array}{c}\text { RES3 } \\
{\left[\mathrm{mV} \mathrm{nm}^{-2}\right]}\end{array}$ & $\begin{array}{c}c_{1} \\
{[\mathrm{mM}]}\end{array}$ & $\begin{array}{c}c_{2} \\
{[\mathrm{mM}]}\end{array}$ & $\begin{array}{c}\varphi \\
{[\mathrm{mV}]}\end{array}$ \\
\hline 0.050 & 20 & 5645.57 & -1600.55 & $2.8310^{-3}$ & 369.291 & 362.685 & 84.022 \\
\hline 0.020 & 25 & 670.06 & -165.69 & $3.1910^{-4}$ & 369.104 & 362.773 & 83.696 \\
\hline 0.010 & 33 & 46.32 & 4.30 & $8.1510^{-6}$ & 368.973 & 362.836 & 83.460 \\
\hline 0.005 & 49 & 13.25 & 3.22 & $5.4710^{-7}$ & 368.942 & 362.851 & 83.398 \\
\hline 0.003 & 71 & 11.98 & -0.69 & $3.7510^{-6}$ & 368.934 & 362.855 & 83.380 \\
\hline
\end{tabular}

from 0.05 to 0.02 resulted in a significant reduction of the residuals, although impact on the discrete values of variables, particularly the concentrations, is less profound. Nevertheless, the calculated time profiles suggest that a satisfactory resolution of the most dynamical phases of the process under study requires values of TOLT below 0.01 .

In addition to the voltage-clamp, an alternative way how to induce an electrodiffusion process in a membrane channel is imposing the electric current of a defined magnitude on the system. We tested also this option using the definition of the right boundary condition as specified in the Theory section (Eq. 8). It should be noted that the boundary condition expressed by Equation 8 prescribes the Neumann condition for the derivative of the potential in accordance with the specified ionic net-flux. Hence, the imposed current represents exclusively the faradaic (conducting) current in contrast to the current-step conditions used previously $[9,13]$ including the displacement current. Since the displacement current appears merely as a transient phenomenon (though possibly of a considerable magnitude) that vanishes in the steady state, it is a matter of the particular problem formulation which current step definition is physically more adequate. In our simulations the NPP system was solved subject to a current step from 0 to $-2 \mathrm{pA} \mathrm{nm}^{-2}$ and spatiotemporal profiles of the ionic concentrations and potential were calculated similarly as for the standard case shown in Figure 3. The faradaic current density of $-2 \mathrm{pA} \mathrm{nm}^{-2}$ developed a steady-state potential of $+168.48 \mathrm{mV}$ at the right boundary of the channel. In order to compare behavior of the system under the voltage and current-clamp conditions, we performed a reference calculation imposing a potential step from 0 to $+168.48 \mathrm{mV}$ at the right channel end. No significant difference in the time courses of the ionic concentrations was observed. The maximum in the concentration of cation is somewhat smaller (difference of $3.5 \mathrm{mmol} \mathrm{dm}^{-3}$ for the current-clamp condition and shifted to a later time (difference of $7.3 \mathrm{~ns}$ ). The anionic concentrations show qualitatively the same sigmoid time course with a similar time shift indicating a longer induction period for the process of charge separation in the current-clamp case. As expected, both simulations attained the same steady state.

The obtained solution of the studied NPP problem in the form of spatiotemporal profiles of the ionic concentrations and the potential provides information on other physical quantities such as the electric field intensity, the ionic fluxes or various components of the electric current. Data for the required quantities as functions of the position and time may be retrieved from the original solution by the numerical differentiation as described in the previous sections. Such data allow deeper insight into the nature of the physical processes underlying the electrodiffusion dynamics in the system under study. A more detailed analysis and interpretation of such processes will be reported separately. As an illustration we present here data concerning the faradaic and displacement currents (Fig. 5). It is evident that the displacement current represents a significant component of the total current, in particular at the channel boundaries. For example, in a time of 1 ns from the voltage jump the displacement current accounts for more than $60 \%$ of the total current magnitude at the position $x=0.25 \mathrm{~nm}$. Despite the spatial inhomogenity of both faradaic and displacement currents, the total current is uniform throughout the channel as it is documented by Figure 5b. This feature of the total current emerges as a consequence of the Poisson and continuity equations as it can be proved by differentiation of the current with respect to $x$ [37]. Verification of spatial uniformity of the total current may serve as another useful test of the reliability of the computed data both with respect to the NPP solution itself and the numerical partial derivatives of the solution. 

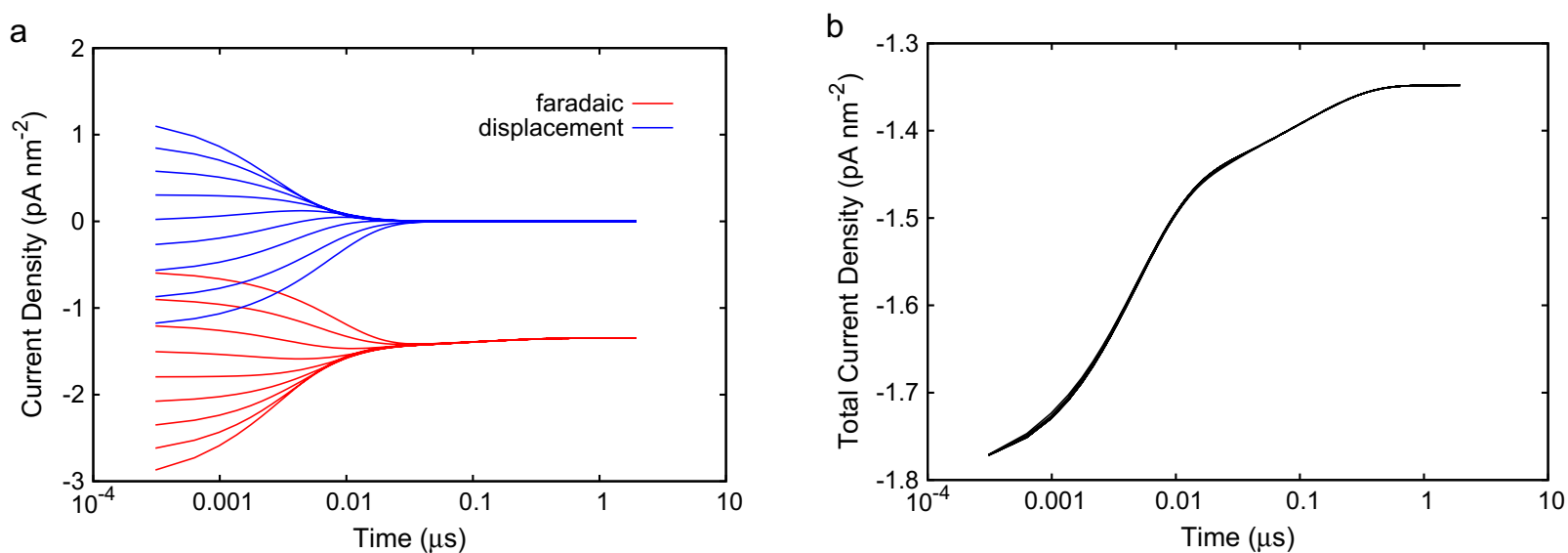

Figure 5 - Time courses of the current density through the channel obtained from the original solution. (a) The faradaic (the lower set of lines - red) and the displacement (the upper set - blue) currents at various equidistant positions starting at $x=0.25 \mathrm{~nm}$ (the top line of the faradaic current set and the bottom line of the displacement current set) and ending at $x=9.75 \mathrm{~nm}$. (b) The total current representing the sum of the corresponding time courses from the panel a. The standard set of parameters was used in the original calculation.

We used the a posteriori numerical partial differentiation also to examine the reliability of the obtained solution in the sense of satisfying the PDEs that were solved. Such an examination is reasonable, since it is known that despite the convergence of the used numerical treatment, in some cases the obtained "solution" may follow an asymptotic solution or a numerical artifact due to the discretization of the problem. For that purpose, we compared the left- and right-hand sides of the solved equations (5)-(7) calculated using the numerical partial derivatives of the obtained solution. Table 2 presents an example of the most critical part of

Table 2 - Comparison of the left-hand (L1-L3) and right-hand (R1-R3) sides of eqs. (5)-(7), respectively for a selected time period at $x=7 \mathrm{~nm}$. The values were calculated by numerical partial differentiation of the standard solution computed on the fixed spatial grid consisting of $160 \times 3$ cells.

\begin{tabular}{|c|c|c|c|c|c|c|}
\hline $\begin{array}{l}\text { Time } \\
{[n s]}\end{array}$ & $\begin{array}{c}\mathrm{L1} \\
{\left[\mathrm{mM} \mathrm{ns}^{-1}\right]^{a}}\end{array}$ & $\begin{array}{c}\mathrm{R} 1 \\
{\left[\mathrm{mM} \mathrm{ns}^{-1}\right]}\end{array}$ & $\begin{array}{c}\mathrm{L} 2 \\
{\left[\mathrm{mM} \mathrm{ns}^{-1}\right]}\end{array}$ & $\begin{array}{c}\mathrm{R} 2 \\
{\left[\mathrm{mM} \mathrm{ns}^{-1}\right]}\end{array}$ & $\begin{array}{c}\mathrm{L3} \\
{\left[\mathrm{mV} \mathrm{nm}^{-2}\right]}\end{array}$ & $\begin{array}{c}\mathrm{R} 3 \\
{[\mathrm{mV} \mathrm{nm}} \\
-2]\end{array}$ \\
\hline 4.931 & 0.1897 & 0.1852 & -0.1152 & -0.1130 & -0.7858 & -0.7858 \\
\hline 5.472 & 0.1321 & 0.1279 & -0.0879 & -0.0859 & -0.8050 & -0.8051 \\
\hline 6.043 & 0.0835 & 0.0796 & -0.0651 & -0.0632 & -0.8193 & -0.8193 \\
\hline 6.646 & 0.0432 & 0.0397 & -0.0462 & -0.0445 & -0.8289 & -0.8289 \\
\hline 7.288 & 0.0106 & 0.0074 & -0.0310 & -0.0295 & -0.8346 & -0.8346 \\
\hline 7.973 & -0.0150 & -0.0178 & -0.0191 & -0.0178 & -0.8366 & -0.8366 \\
\hline 8.709 & -0.0343 & -0.0368 & -0.0102 & -0.0090 & -0.8355 & -0.8355 \\
\hline 9.505 & -0.0480 & -0.0502 & -0.0039 & -0.0029 & -0.8317 & -0.8317 \\
\hline 10.372 & -0.0567 & -0.0586 & 0.0001 & 0.0009 & -0.8257 & -0.8257 \\
\hline 11.325 & -0.0612 & -0.0628 & 0.0021 & 0.0028 & -0.8178 & -0.8178 \\
\hline 12.383 & -0.0622 & -0.0634 & 0.0025 & 0.0030 & -0.8085 & -0.8086 \\
\hline 13.570 & -0.0603 & -0.0611 & 0.0016 & 0.0019 & -0.7983 & -0.7983 \\
\hline 14.919 & -0.0562 & -0.0567 & -0.0004 & -0.0002 & -0.7874 & -0.7874 \\
\hline 16.471 & -0.0508 & -0.0509 & -0.0031 & -0.0030 & -0.7765 & -0.7765 \\
\hline 18.279 & -0.0447 & -0.0445 & -0.0061 & -0.0062 & -0.7659 & -0.7659 \\
\hline 20.405 & -0.0386 & -0.0382 & -0.0093 & -0.0095 & -0.7561 & -0.7561 \\
\hline 22.914 & -0.0334 & -0.0328 & -0.0124 & -0.0126 & -0.7475 & -0.7476 \\
\hline
\end{tabular}


the solution that covers time period approximately from 5 to $23 \mathrm{~ns}$ for the position $x=7 \mathrm{~nm}$. The respective sides of the NPP equations compare very well except in a few points. For example, the $\mathrm{L} 1$ and $\mathrm{R} 1$ values significantly differ at the time of $7.288 \mathrm{~ns}$. However, one should consider that in this particular point the examined values are close to zero as the time derivative of the cationic concentration change the sign. Hence, it may be assumed that the concerned numerical derivative carries a high relative error which is probably the cause of the observed discrepancy.

We can conclude that the adopted numerical technique for solving the time-dependent Nernst-Planck and Poisson equations shows numerical reliability and efficiency together with "well posedness" and structural stability in terms introduced by Baxter \& Iserles [38]. The technique proved to be a useful tool, potentially capable of resolving electrodiffusion dynamics of quite complex models in higher spatial dimensions.

\section{ACKNOWLEDGMENTS}

This work was supported by the European Science Foundation program FuncDyn (short visit grant No. 2283 for I.V.) and Slovak Research and Development Agency under the contract No. APVV0059-10. Thanks are due to Rui Dilão for discussion on numerical solving of PDEs and Roman Jartim for help with searching for the older literature.

\section{REFERENCES}

[1] PLANCK M. 1889. Ann. Physik. Chem. N.F., 39: 161.

[2] GOLDMAN DE. 1943. Journal of General Physiology, 27: 37-60.

[3] MAFÉ S, PELLICER J \& AGUILELLA VM. 1986. Journal of Physical Chemistry, 90: 6045-6050.

[4] BARCILON V, CHEN D-P \& EISENBERG RS. 1992. SIAM Journal on Applied Mathematics, 52: 1405-1425.

[5] KURNIKOVA MG, COALSON RD, GRAF P \& NITZAN A. 1999. Biophysical Journal, 76: 642-656.

[6] RAMIREZ P, MAFÉ S, AGUILELLA VM \& ALCARAZ A. 2003. Physical Review E, 68: 011910.

[7] LIU Q, WANG Y, GUO W, JI H, XUE J \& OUYANG Q. 2007. Physical Review E, 75: 051201.

[8] HELFFERICH F \& PLESSET MS. 1958. Journal of Chemical Physics, 28: 418-424.

[9] COHEN H \& COOLEY JW. 1965. Biophysical Journal, 5: 145-162.

[10] HAFEMANN DR. 1965. Journal of Physical Chemistry, 69: 42264231.
[11] MacGILLIVRAY AD. 1970. Journal of Chemical Physics, 52: 3126-3132.

[12] OFFNER F. 1974. Journal of Theoretical Biology, 45: 81-91.

[13] BRUMLEVE TR \& BUCK RP. 1978. Journal of Electroanalytical Chemistry, 90: 1-13.

[14] MURPHY WD, MANZANARES JA, MAFÉ S \& REISS H. 1992. Journal of Physical Chemistry, 96: 9983-9991.

[15] MANZANARES JA, MURPHY WD, MAFÉ S \& REISS H. 1993. Journal of Physical Chemistry, 97: 8524-8530.

[16] RUDOLPH M. 1994. Journal of Electroanalytical Chemistry, 375: 89-99.

[17] SOKALSKI T, LINGENFELTER P \& LEWENSTAM A. 2003. Journal of Physical Chemistry, 107: 2443-2452.

[18] BIENIASZ LK. 2004. Journal of Electroanalytical Chemistry, 565: 251-271.

[19] LIM J, WHITCOMB J, BOYD J \& VARGHESE J. 2007. Journal of Colloid and Interface Science, 305: 159-174.

[20] DAS S, DUBSKY P, BERG A \& EIJKEL JCT. 2012. Physical Review Letters, 108: 138101.

[21] WANG H \& PILON L. 2012. Electrochimica Acta, 64: 130-139.

[22] GRYSAKOWSKI B, JASIELEC JJ, WIERZBA B, SOKALSKI T, LEWENSTAM A \& DANIELEWSKI M. 2011. Journal of ElectroanaIytical Chemistry, 662: 143-149.

[23] SAMSON E \& MARCHAND J. 1999. Journal of Colloid and Interface Science, 215: 1-8.

[24] OLESEN LH, BAZANT MZ \& BRUUS H. 2010. Physical Review E, 82: 011501.

[25] PAZ-GARCía JM, JOHANNESSON B, OTTOSEN LM, RIBEIRO AB \& RODRÍGUES-MAROTO JM. 2011. Separation and Purification Technology, 79: 183-192.

[26] HORNO J, CASTILLA J \& GONZÁLES-FERNÁNDES CF. 1992. Journal of Physical Chemistry, 96: 854-858.

[27] SAMSON E, MARCHAND J, ROBERT J-L \& BOURNAZEL J-P. 1999. International Journal for Numerical Methods in Engineering, 46: 2043-2060.

[28] BAZANT MZ, THORNTON K \& AJDARI A. 2004. Physical Review E, 70: 021506.

[29] SOESTBERGEN M, BIESHEUVEL PM \& BAZANT MZ. 2010. Physical Review E, 81: 021503.

[30] BEUNIS F, STRUBBE F, MARESCAUX M, BEECKMAN J, NEYTS K \& VERSCHUEREN ARM. 2008. Physical Review E, 78: 011502.

[31] GOLOVNEV A \& TRIMPER S. 2009. Journal of Chemical Physics, 131: 114903. 
[32] BLOM JG, TROMPERT RA \& VERWER JG. 1996. ACM Transactions on Mathematical Software, 22: 302-328.

[33] ZHOU SA \& UESAKA M. 2009. International Journal of Applied Electromagnetics and Mechanics, 29: 25-36.

[34] HERAS JA. 2011. American Journal of Physics, 79: 409-416.

[35] KEENER J \& SNEYD J. 1998. Mathematical Physiology, 82-87. Springer-Verlag, New York.
[36] CORRY B, KUYUCAK S \& CHUNG S-H. 2000. Biophysical Journal, 78: 2364-2381.

[37] ARNDT RA \& ROPER LD. 1973. Mathematical Biosciences, 16: 103-117.

[38] BAXTER BJC \& ISERLES A. 2003. In: CUCKER F (Ed.) Special Volume: Foundations of Computational Mathematics, 3-34. Elsevier Science B.V. 